PROCEEDINGS OF THE

AMERICAN MATHEMATICAL SOCIETY

Volume 130, Number 9, Pages 2593-2598

S 0002-9939(02)06624-8

Article electronically published on April 17, 2002

\title{
CHEBYSHEV CENTRES AND CENTRABLE SETS
}

\author{
T. S. S. R. K. RAO \\ (Communicated by Jonathan M. Borwein) \\ Dedicated to the memory of my father
}

\begin{abstract}
In this paper we characterize real Banach spaces whose duals are isometric to $L^{1}(\mu)$ spaces (the so-called $L^{1}$-predual spaces) as those spaces in which every finite set is centrable. For a locally compact, non-compact set $X$ and for an $L^{1}$-predual $E$, we give a complete description of the extreme points and denting points of the dual unit ball of $C_{0}(X, E)$, equipped with the diameter norm.
\end{abstract}

\section{INTRODUCTION}

Let $E$ be a Banach space. A bounded set $A \subset E$ is said to be centrable if $\frac{1}{2} \operatorname{dia}(A)=\inf _{e \in E} \sup _{a \in A}\|a-e\|$ (where $\operatorname{dia}(A)$ stands for the diameter of the set $A$ ). The number on the right-hand side is called the Chebyshev radius of $A$ and is always bigger than or equal to half the diameter (see [H], pages 177 and 192]). $e_{0} \in E$ is said to be a Chebyshev centre of $A$ if inf $f_{e \in E} \sup _{a \in A}\|a-e\|=$ $\sup _{a \in A}\left\|a-e_{0}\right\|$. Chebyshev centres and centrable sets are important concepts in the theory of optimization. It is known that in a dual space any bounded set has a Chebyshev centre. It was remarked in [BR] (see Corollary 3.4) that any finite set in an $L^{1}$-predual space has a Chebyshev centre. When the scalar field is real, for any compact extremally disconnected set $X$, every bounded set in $C(X)$ is centrable (see [H, page 193]). Motivated by a recent characterization of the space $C(X)$ in terms of relative Chebyshev radius obtained in [E], in the second section of this paper we study real Banach spaces $E$ in which finite sets have the same Chebyshev radius in all superspaces. It is well-known that the dual of $C(X)$ is isometric to an $L^{1}(\mu)$ space; see [L, Chapter 1]. Thus $L^{1}$-preduals appear to be the right spaces for studying questions related to these concepts. We first characterize real Banach spaces in which every finite set is centrable as those whose duals are isometric to $L^{1}(\mu)$-spaces. This allows us to characterize real $L^{1}$-predual spaces as those Banach spaces $E$ in which finite sets have the same Chebyshev radius in all isometric embedding of $E$ into another real Banach space $F$.

Let $X$ be a locally compact, non-compact Hausdorff space and let $C_{0}(X, E)$ denote the space of $E$-valued continuous functions on $X$ that vanish at infinity over the real scalar field. For any $f \in C_{0}(X, E)$, let $\|f\|_{\rho}=\operatorname{dia}(f(X))$. This is a norm on $C_{0}(X, E)$. Our main result is the identification of the extreme points

Received by the editors February 12, 2001.

2000 Mathematics Subject Classification. Primary 41A65, 46B20.

Key words and phrases. Chebyshev centre, centrable set, diameter norm.

(C)2002 American Mathematical Society 
and denting points of the dual unit ball of this space when $E$ is a real $L^{1}$-predual space. Let $E_{1}$ denote the closed unit ball of $E$. We denote by $\partial_{e} E_{1}$ the set of extreme points of $E_{1}$. For any $x \in X$ and $e^{*} \in \partial_{e} E_{1}^{*}$ we denote by $\epsilon_{x} \otimes e^{*}$ the functional $\epsilon_{x} \otimes e^{*}(f)=e^{*}(f(x))$. With this notation our result states that $\partial_{e}\left(C_{0}(X, E)\right)_{1}^{*}=\left\{\epsilon_{x} \otimes e^{*},\left(\epsilon_{x}-\epsilon_{y}\right) \otimes e^{*}: x \neq y \in X\right.$ and $\left.e^{*} \in \partial_{e} E_{1}^{*}\right\}$. Such a description in the case of scalar-valued functions was given in $[\mathrm{C}$ when $X$ is compact and in $[\mathrm{FS}$ ] for locally compact $X$. Our proof is much simpler than the ones given in $\mathrm{C}]$ and $[\mathrm{FS}]$.

A linear surjective map $\Phi: C_{0}(X, E) \rightarrow C_{0}(X, E)$ is said to be diameterpreserving if for any $f \in C_{0}(X, E)$, dia $(f(X))=\operatorname{dia}(\Phi(f)(X))$. Note that w.r.t. the diameter norm $\Phi$ is an onto isometry. In order to obtain a description of these maps, as in the case of the proof of the classical Banach-Stone theorem that describes the onto isometries w.r.t. the supremum norm, obtaining a description of the set of extreme points of the dual unit ball is a central step. When $E$ is an $L^{1}$-predual space, we show that there is an onto homeomorphism $\phi$ of $X$ and an onto isometry $S$ of $E$ such that $\Phi(f)(x)=S(f(\phi(x)))$.

In the concluding section of this paper we turn to Banach spaces over the complex scalar field. It is easy to construct a set of 3 elements in the complex plane that is not centrable. In order to overcome this difficulty, we define a bounded set $A$ to be weakly centrable if for every $e^{*} \in E_{1}^{*}, e^{*}(A)$ is a centrable subset of the complex plane. Since any bounded set of real numbers is centrable, this notion is redundant in spaces over the real scalar field. It is easy to see that any weakly centrable finite set of complex numbers is centrable. In the last part of this paper, we show that when the scalar field is complex, for any compact extremally disconnected space $X$, every bounded weakly centrable set in $C(X)$ is centrable. This allows us to give a correct formulation of Theorem 3.9 in $\mathrm{BR}$ by showing that in a complex $L^{1}$-predual space every weakly centrable compact set is centrable.

Our notation and terminology is standard and can be found in $[\mathrm{H}]$ and $[\mathrm{DU}]$. For a compact set $X$, we always consider the supremum norm on the space $C(X, E)$. This work was done during the year 1999-2000 when the author had a visiting position at the University of Missouri-Columbia. The author is grateful to the Department of Mathematics at MU for their warm hospitality.

\section{A Characterization OF $L^{1}$-PREDUAL SPACES AND SOME APPLICATIONS}

Throughout this section we assume that the scalar field is real.

Let $E \subset F$ be Banach spaces. For any bounded set $A \subset E$, since the diameter is the same in both $E$ and $F$, if it is centrable in $E$, then it is also centrable in $F$. When $A$ is considered as a subset of $F$, we in particular have that the relative Chebyshev radius (w.r.t. $E$ ) of $A$ in $F$ is the same as its Chebyshev radius. We next consider the question "when every finite set in $E$ that is centrable in $F$ is also centrable in E?" We introduce a definition along the lines of a "central subspace" defined in $[\mathrm{BR}$.

Definition. $E$ is said to be an almost central subspace of $F$ if for any finite set $\left\{e_{1}, \ldots, e_{n}\right\} \subset E$, for any $f \in F$ and $\epsilon>0$, there exists an $e \in E$ (possibly depending on $f$ and $\epsilon$ ) such that

$$
\max _{1 \leq i \leq n}\left\|e-e_{i}\right\| \leq \max _{1 \leq i \leq n}\left\|f-e_{i}\right\|+\epsilon
$$


Lemma. Let $E \subset F$ be an almost central subspace. Any finite subset of $E$ that is centrable in $F$ is also centrable in $E$.

Proof. Let $\left\{e_{1}, \ldots, e_{n}\right\} \subset E$ be a centrable set in $F$. As remarked before we only need to show that the relative Chebyshev radius of this set is less than or equal to its Chebyshev radius. Let $f \in F$ and let $\epsilon>0$. By our assumption there exists an $e_{0} \in E$ such that $\max _{1 \leq i \leq n}\left\|e_{0}-e_{i}\right\| \leq \max _{1 \leq i \leq n}\left\|f-e_{i}\right\|+\epsilon$. Therefore

$$
\text { inf }_{e \in E} \max _{1 \leq i \leq n}\left\|e-e_{i}\right\| \leq \max _{1 \leq i \leq n}\left\|f-e_{i}\right\|+\epsilon .
$$

Hence $i n f_{e \in E} \max _{1 \leq i \leq n}\left\|e-e_{i}\right\| \leq i n f_{f \in F} \max _{1 \leq i \leq n}\left\|f-e_{i}\right\|$.

Theorem 1. Let $E$ be a real Banach space. TFAE.

1. Every finite set is centrable in $E$.

2. $E$ is an almost central subspace of any superspace $F$.

3. $E$ is isometric to an $L^{1}$-predual space.

Proof. $1 \Rightarrow 2$. Suppose $E$ is a real Banach space such that every finite set is centrable. Let $E \subset F$. Let $\left\{e_{1}, \ldots, e_{n}\right\} \subset E, f \in F$ and $\epsilon>0$. Since $\left\{e_{1}, \ldots, e_{n}\right\}$ is centrable in $F$, we have that the Chebyshev radii agree and thus

$$
\text { inf }_{e \in E} \max _{1 \leq i \leq n}\left\|e-e_{i}\right\|<\max _{1 \leq i \leq n}\left\|f-e_{i}\right\|+\epsilon .
$$

Therefore there exists an $e \in E$ such that $\max _{1 \leq i \leq n}\left\|e-e_{i}\right\| \leq \max _{1 \leq i \leq n}\left\|f-e_{i}\right\|$.

$2 \Rightarrow 1$. It is well known that $E$ is isometric to a subspace of $\ell^{\infty}\left(E_{1}^{*}\right)$ where $E_{1}^{*}$ is equipped with the discrete topology. Also the latter space is isometric to a $C(X)$ for a compact extremally disconnected space $X$. In this space we know that finite sets are centrable. Therefore it follows from our Lemma that any finite set is centrable in $E$.

$3 \Rightarrow 2$. If $E$ is an $L^{1}$-predual space, it follows from Theorem 3.3 of $B$, that $E$ is in fact a central subspace of any superspace.

$2 \Rightarrow 3$. It follows from the proof of the characterization of $L^{1}$-preduals in terms of intersection properties of balls (see Theorem 6 on page 212 and Lemma 3 on page 208 of $\left(\mathrm{L}\right.$ ) that $E$ is an $L^{1}$-predual, if and only if for every $r, \epsilon>0$ and for any finite collection $\left\{B\left(a_{i}, r\right)\right\}_{1 \leq i \leq n}$ of pairwise intersecting closed balls, $\bigcap_{1 \leq i \leq n} B\left(a_{i}, r+\epsilon\right) \neq$ $\emptyset$. Embedding $E$ into $\ell^{\infty}\left(E_{1}^{*}\right)$ as before and noting that the latter space is an $L^{1}$ predual, it is easy to see that $2 \Rightarrow 3$.

Corollary 1. Let $E$ be a real Banach space. If every finite set is centrable in E, then every finite set has a Chebyshev centre.

Proof. We have from the above theorem that $E$ is an $L^{1}$-predual. Therefore it follows from Corollary 3.4 in $[\mathrm{BR}]$ that any finite set has a Chebyshev centre.

Corollary 2. Let $E$ be a real Banach space. $E$ is an $L^{1}$-predual iff every finite set has the same Chebyshev radius in all superspaces of $E$.

Proof. Suppose every finite set in $E$ has the same Chebyshev radius in all superspaces of $E$. Since $E$ is isometric to a subspace of $\ell^{\infty}\left(E_{1}^{*}\right)$, as before we conclude that any finite subset of $E$ is centrable and hence $E$ is an $L^{1}$-predual.

Conversely suppose that $E$ is an $L^{1}$-predual. Since finite sets are centrable in $E$ it follows from the remarks made earlier that finite subsets of $E$ have the same Chebyshev radius in all superspaces of $E$. 
Remark 1. Using an $\epsilon$-net argument it is easy to see that if every finite set in $E$ is centrable, then every compact set is centrable.

We now turn to the description of extreme and denting points of $C_{0}(X, E)_{1}^{*}$ endowed with the diameter norm $\|.\|_{\rho}$. It is possible to approach this question using injective tensor products. However we prefer to use an identification due to Singer (see [DU]) of the dual of the space of vector-valued continuous functions on a compact set as the space of vector measures with the bounded variation norm. Thus essential to our arguments below is a precise identification of the diameter norm on $C_{0}(X, E)$ in terms of the supremum norm on a space of $E$-valued functions on a compact set. Let $X^{*}$ denote the one point compactification of $X$. For any $e \in E$, by $1 \otimes e$ we denote the constant function whose value is $e$ in $C\left(X^{*}, E\right)$. By $1 \otimes E$ we denote the closed span of the constant functions.

Theorem 2. Let $E$ be an $L^{1}$-predual space. $\partial_{e} C_{0}(X, E)_{1}^{*}=\left\{\epsilon_{x} \otimes e^{*},\left(\epsilon_{x}-\epsilon_{y}\right) \otimes e^{*}\right.$ : $\left.x \neq y \in X, e^{*} \in \partial_{e} E_{1}^{*}\right\}$.

Proof. For any $f \in C_{0}(X, E)$, let $f^{\prime} \in C\left(X^{*}, E\right)$ denote its natural extension. Define $\Phi: C_{0}(X, E) \rightarrow C\left(X^{*}, E\right) / 1 \otimes E$ by $\Phi(f)=\left[f^{\prime}\right]$ ([.] denotes the equivalence class). Clearly $\Phi$ is linear. We recall that the space $C\left(X^{*}, E\right)$ is equipped with the supremum norm. Since $f^{\prime}\left(X^{*}\right)=\overline{f(X)}$ and since compact sets are centrable, we have $\frac{1}{2}\|f\|_{\rho}=\frac{1}{2} \operatorname{dia}(f(X))=\frac{1}{2} \operatorname{dia}\left(f^{\prime}\left(X^{*}\right)\right)=\inf _{e \in E} s u p_{x \in X^{*}}\left\|e-f^{\prime}(x)\right\|=$ in $f_{e \in E}\left\|1 \otimes e-f^{\prime}\right\|=\left\|\left[f^{\prime}\right]\right\|$. Also for any $g \in C\left(X^{*}, E\right)$, let $e=g(\infty)$. Since $g-1 \otimes e$ vanishes at infinity and $[g]=[g-1 \otimes e]$, we see that $\Phi$ is an onto map.

By a well-known result of Singer (see [DU]), $C\left(X^{*}, E\right)^{*}$ can be identified with the space of $E^{*}$-valued countably additive regular Borel measures of finite variation, equipped with the total variation norm. Thus the norm on $C_{0}(X, E)^{*}$ is half the total variation norm on the space of vector measures vanishing on $1 \otimes E$. Note that for any unit vector $e^{*} \in E^{*}$ and $x \neq y \in X^{*},\left\|\left(\epsilon_{x}-\epsilon_{y}\right) \otimes e^{*}\right\|_{\rho}^{*}=1$ since the measure $\left(\epsilon_{x}-\epsilon_{y}\right) \otimes e^{*}$ vanishes on $1 \otimes E$ and its total variation norm is 2 .

For any $x \neq y \in X^{*}$ and for any $e^{*} \in \partial_{e} E_{1}^{*}$, we now show that $\left(\epsilon_{x}-\epsilon_{y}\right) \otimes e^{*}$ is an extreme point of the two balls of the space of vector measures vanishing on $1 \otimes E$, w.r.t. the total variation norm. Note that when $y=\infty,\left(\epsilon_{x}-\epsilon_{y}\right) \otimes e^{*}=\epsilon_{x} \otimes e^{*}$ on $C_{0}(X, E)$.

Suppose $\left(\epsilon_{x}-\epsilon_{y}\right) \otimes e^{*}=\frac{1}{2}\{F+G\}$ with $\|F\|=\|G\|=2$. Clearly $F, G$ are supported on $\{x, y\}$. Hence $2=\|F(x)\|+\|F(y)\|=\|G(x)\|+\|G(y)\|$. Also $e^{*}=\frac{1}{2}\{F(x)+G(x)\}$ and $-e^{*}=\frac{1}{2}\{F(y)+G(y)\}$. Moreover,

$$
\begin{aligned}
2 & =\frac{1}{2}\|F+G\| \leq \frac{1}{2}\{\|F(x)+G(x)\|+\|F(y)+G(y)\|\} \\
& \leq \frac{1}{2}\{\|F(x)\|+\|F(y)\|+\|G(x)\|+\|G(y)\|\}=2 .
\end{aligned}
$$

Since $e^{*}$ and $-e^{*}$ are extreme points we get that $\left(\epsilon_{x}-\epsilon_{y}\right) \otimes e^{*}$ is an extreme point.

Let $F \in \partial_{e} C_{0}(X, E)_{1}^{*}$. Consider the mapping $\Psi: C_{0}(X, E) \rightarrow C\left(X^{*} \times X^{*}, E\right)$ defined by $\Psi(f)(x, y)=f^{\prime}(x)-f^{\prime}(y)$. We recall once again that the space $C\left(X^{*} \times\right.$ $\left.X^{*}, E\right)$ is equipped with the supremum norm. It is easy to see that $\Psi$ is an isometry. Using a lemma of Singer $([\underline{\mathrm{H}}$, page 82$])$ we can transfer and extend $F$ to an extreme point of $C\left(X^{*} \times X^{*}, E\right)_{1}^{*}$. Since extreme points of this latter set are given by Dirac measures at extreme points of $E_{1}^{*}$, we get the desired conclusion. 
Corollary 3. Let $E$ be an $L^{1}$-predual space. The denting points of $C_{0}(X, E)_{1}^{*}$ are precisely those extreme points for which $e^{*}$ is a denting point of $E_{1}^{*}$.

Proof. Let $e^{*} \in E_{1}^{*}$ be a denting point. In view of the above theorem we need to show that for any $x \neq y \in X^{*},\left(\epsilon_{x}-\epsilon_{y}\right) \otimes e^{*}$ is a denting of the two balls in the space of vector measures vanishing on $1 \otimes E$. Since it is an extreme point, using the results from [LLT], we need to prove that it is a point of weak-norm continuity in the two balls, which is already shown in [HS, Proposition 11]. The converse statement is similarly proven using the results of [LLT] and once again Proposition 11 from [HS].

We now describe the isometries of $C_{0}(X, E)$ when $E$ is an $L^{1}$-predual space, equipped with the diameter norm. A description in the case of $C(X, E)$ when $X$ is a compact set was given in $[\mathrm{RR}]$. A key factor of the proof in $[\mathrm{RR}$ is the knowledge of the extreme points of the dual unit ball of the space of vector-valued functions. The following proposition follows from Theorem 2 and arguments similar to the ones given during the proof of Theorem 2 in [RR].

Proposition 1. Let $\Phi$ be any onto isometry of $C_{0}(X, E)$. There exists an onto homeomorphism $\phi$ of $X$ and an onto isometry $S$ of $E$ such that $\Phi(f)(x)=S(f(\phi(x)))$.

Proof. Define $\Phi^{\wedge}: C\left(X^{*}, E\right) / 1 \otimes E \rightarrow C\left(X^{*}, E\right) / 1 \otimes E$ by

$$
\Phi^{\wedge}([f])=[\Phi(f-1 \otimes f(\infty))] .
$$

Then $\Phi^{\wedge}$ is a linear bi-continuous bijection. Since $E$ is an $L^{1}$-predual space, for any $e^{*} \in \partial_{e} E_{1}^{*}$, there is a closed subspace $N$ of $E^{*}$ such that $E^{*}$ is the $\ell^{1}$-direct sum of $\operatorname{span}\{e\}$ and $N$. Thus for $x \neq y$ and $e_{1}^{*} \neq \pm e_{2}^{*}$, the total variation norm of $\left(\epsilon_{x} \otimes e_{1}^{*}+\epsilon_{y} \otimes e_{2}^{*}\right)$ is 2 . It can now be proved using the arguments given during the proof of Theorem 2 in $\left[\mathrm{RR}\right.$ that there is a homeomorphism $\phi$ of $X^{*}$ and an onto isometry $S$ of $E$ such that $\Phi(f)(x)=S(f(\phi(x)))$.

\section{Complex Banach spaces and Centrability}

Our first result is the complex version of the Theorem on page $193 \mathrm{in}[\mathrm{H}$. This allows us to give a correct formulation of Theorem 3.9 in [BR] by showing that in a complex $L^{1}$-predual space every weakly centrable compact set is centrable.

We recall from $[\mathrm{Hu}]$ that for any index set $\Gamma$, a family $\left\{B\left(e_{\gamma}, r_{\gamma}\right)\right\}_{\gamma \in \Gamma}$ of closed balls is said to have the weak intersection property if $\bigcap_{\gamma \in \Gamma} B\left(e^{*}\left(e_{\gamma}\right), r_{\gamma}\right) \neq \emptyset$ for all $e^{*} \in E_{1}^{*}$. The main result of $[\mathrm{Hu}]$ implies that a complex Banach space $E$ is isometric to a $C(X)$ for some compact extremally disconnected space iff every weakly intersecting family of closed balls has non-empty intersection.

Proposition 2. Let $X$ be a compact extremally disconnected space and let $E=$ $C(X)$. Any bounded weakly centrable set in $E$ is centrable.

Proof. Let $A \subset E$ be a bounded weakly centrable set. Let $r=\frac{1}{2} \operatorname{dia}(A)$. We claim that the family of balls $\{B(a, r)\}_{a \in A}$ has the weak intersection property. To see this, let $e^{*} \in E_{1}^{*}$. Since $\left\{e^{*}(A)\right\}$ is a centrable set and since it has a Chebyshev centre, there exists an $\alpha_{0}$ such that $\sup _{a \in A}\left|\alpha_{0}-e^{*}(a)\right|=\frac{1}{2} \operatorname{dia}\left(e^{*}(A)\right) \leq \frac{1}{2} \operatorname{dia}(A)=r$. Thus by Hustad's result $\left[\mathrm{Hu}\right.$, there exists an $e_{0} \in E$ such that $S u p_{a \in A}\left\|e_{0}-a\right\| \leq$ $r=\frac{1}{2} \operatorname{dia}(A)$. Therefore $A$ is centrable.

Corollary 4. Let $E$ be a complex $L^{1}$-predual space. Every weakly centrable compact set in $E$ is centrable. 
Proof. Let $K \subset E$ be a weakly centrable compact set. Consider the canonical embedding $E \subset E^{* *}$. Since for any $\Lambda \in E^{* * *}, \Lambda(K)=(\Lambda \mid E)(K)$, we can see that $K$ is a weakly centrable set in $E^{* *}$. Since $E^{* *}$ is isometric to $C(X)$ for a compact extremally disconnected set $X$ (see $[\mathrm{L}$, sections 11 and 23]), it follows from the above proposition that $K$ is a centrable set in $E^{* *}$. Also when $K$ is considered as a subset of the dual space $E^{* *}$ it has a Chebyshev centre in $E^{* *}$. Now arguments similar to the ones given during the proof of Theorem 3.19 in [BR] (those arguments work in either scalar field) give the desired conclusion.

Remark 2. Let $\left\{\alpha_{1}, \alpha_{2}, \alpha_{3}\right\}$ be any non-centrable set. For a sufficiently large $r>0$, we have $\bigcap_{1 \leq i \leq 3} B\left(\alpha_{i}, r\right) \neq \emptyset$. Thus $\left\{B\left(\alpha_{i}, r\right)\right\}_{1 \leq i \leq 3}$ has the weak intersection property but the centres do not form a centrable (weakly centrable) set. Thus for the complex scalar field we are unable to classify intersection properties of balls in terms of centrability and Chebyshev centres.

\section{REFERENCES}

[BR] P. Bandyopadhyay and T. S. S. R. K. Rao, Central subspaces of Banach spaces, J. Approx. Theory, 103 (2000) 206-222. MR 2001b:46022

[C] F. Cabello Sanchez, Diameter preserving linear maps and isometries, Arch. Math., 73(1999) 373-379. MR 2000j:46047

[DU] J. Diestel and J. J. Uhl, Vector measures, AMS Surveys No 15, Providence, RI, 1977. MR 56:12216

[E] R. Espínola, A. Wiśnicki and J. Wośko, A geometrical characterization of the $C(K)$ and $C_{0}(K)$ spaces, J. Approx. Theory, 105 (2000) 87-101. MR 2001g:46026

[FS] J. J. Font and M. Sanchis, A characterization of locally compact spaces with homeomorphic one point compactification, Top. Appl., to appear.

$[\mathrm{H}] \quad$ R. B. Holmes, A course on optimization and best approximation, LNM No 257, SpringerVerlag, Berlin, 1972. MR 54:8381

[HS] Z. Hu and M. Smith, On the extremal structure of the unit ball of the space $C(K, X)^{*}$, in: Proc. Conf. on Function Spaces (SIUE), Lecture Notes in Pure and Appl. Math. No 172, Marcel Dekker, 1995, 205-223. MR 96k:46062

[Hu] O. Hustad, A note on complex $\mathcal{P}_{1}$-spaces, Israel J. Math., 16 (1973) 117-119. MR 48:9351

[L] H. E. Lacey, Isometric theory of classical Banach spaces, Grundlehren Math. Wiss., Band 208, Springer-Verlag, Berlin, 1973. MR 58:12308

[LLT] B. L. Lin, P. K. Lin and S. L. Troyanski, Characterizations of denting points, Proc. Amer. Math. Soc., 102 (1988) 526-528. MR 89e:46016

[RR] T. S. S. R. K. Rao and A. K. Roy, Diameter-preserving linear bijections of function spaces, J. Austral. Math. Soc., 70 (2001) 323-335. MR 2002b:46039

Indian Statistical Institute, R. V. College Post, Bangalore-560059, India

E-mail address: tss@isibang.ac.in 